\title{
A Medicina Remota em Tempos de Pandemia: um estudo prospectivo de tecnologias de telemedicina
}

\author{
Remote Medicine in Pandemic Times: a prospective study of \\ telemedicine technologies
}

\author{
Felipe Roberto Eloi Moura ${ }^{1}$ \\ Kaline Silva dos Santos ${ }^{1}$ \\ Ailton Mota do Nascimento Galvão ${ }^{1}$ \\ Guilherme Benjamin Brandão Pitta ${ }^{1}$ \\ ${ }^{1}$ Universidade Federal de Alagoas, Maceió, AL, Brasil
}

\begin{abstract}
Resumo
A telemedicina abrange atividades médicas, como diagnóstico, tratamento, prevenção de doenças e educação continuada, se utilizando principalmente de tecnologias de informação, imagem de vídeo e conexões de telecomunicações, proporcionando tratamento remoto, monitoramento contínuo e compartilhamento de informações. Essa abordagem da assistência médica tem sido especialmente importante diante da pandemia da Covid-19 que o mundo ainda atravessa devido aos seus inúmeros benefícios. Esta pesquisa se propôs a realizar um estudo de prospecção tecnológica em base de patentes, a fim de investigar tecnologias de telemedicina. Foi realizado um levantamento patentário na base de patentes Questel Orbit. Com os dados apurados, análises foram realizadas para destacar países, empresas, classificações de patentes e o atual cenário do Brasil em termos de tecnologias de telemedicina. Notou-se um crescimento considerável de depósitos de patentes nos últimos dez anos, sendo China, Estados Unidos e Coreia do Sul os países que mais patenteiam essas tecnologias.
\end{abstract}

Palavras-chave: Telemedicina. Covid-19. Patentes.

\begin{abstract}
Telemedicine encompasses medical activities such as diagnosis, treatment, disease prevention and continuing education, using mainly information technology, video image and telecommunications connections, providing remote treatment, continuous monitoring and information sharing. This approach to health care has been especially important in the face of the covid-19 pandemic that the world is still going through, due to its numerous benefits. This research focused on conducting a technological prospecting study on the basis of patents, in order to investigate telemedicine technologies. A patent survey was carried out on the basis of Questel Orbit patents. With the data collected, analyzes were carried out to highlight countries, companies, patent classifications and the current scenario in Brazil in terms of telemedicine technologies. There has been a considerable increase in patent filings in the last ten years, with China, the United States and South Korea being the countries that most patent these technologies.
\end{abstract}

Keywords: Telemedicine. Covid-19. Patents.

Área Tecnológica: Tecnologia Médica. Prospecção Tecnológica. 


\section{Introdução}

As Tecnologias da Informação e Comunicação (TICs) possuem um grande potencial no enfrentamento de desafios por países desenvolvidos e em desenvolvimento na questão do fornecimento de serviços de saúde, desafios que passam por acessibilidade, economia e alta qualidade. Segundo a Organização Mundial da Saúde (OMS, 2010), a telemedicina usa as TICs para superar as barreiras geográficas e aumentar o acesso aos serviços de saúde. Isso é particularmentebenéfico para as comunidades rurais e carentes em países em desenvolvimento - grupos que tradicionalmente sofrem com a falta de acesso a cuidados de saúde.

A telemedicina engloba toda a gama de atividades médicas - incluindo diagnóstico, tratamento e prevenção de doenças, educação continuada de profissionais de saúde e consumidores - e pesquisa e avaliação. Em suma, a telemedicina é a prestação de cuidados de saúde e a troca de informações sobre cuidados de saúde a distância (CRAIG; PATTERSON, 2005). Tirando proveito das tecnologias da informação, das imagens de vídeo e das conexões de telecomunicações, a telemedicina possibilita que os médicos forneçam serviços de saúdes a distância (WEINSTEIN et al., 2014).

De acordo com Kvedar, Coye e Everett (2014), é crescente o consenso de estudos que demonstram que as tecnologias de saúde conectadas podem tornar a assistência médica mais eficaz e eficiente ao conectar eletronicamente médicos a médicos, pacientes a médicos e até mesmo pacientes a outros pacientes. Dessa forma, o diagnóstico e o tratamento remoto, o monitoramento contínuo e o ajuste das terapias, o suporte para autocuidado do paciente e o aproveitamento de provedores em grandes populações de pacientes podem ser facilitados com essa abordagem. Ademais, essas tecnologias podem melhorar o compartilhamento de dados e tarefas entre as equipes, permitindo que os membros pratiquem em seus níveis mais altos de habilidade e treinamento.

Na literatura também há muitas discussões sobre as diferenças e semelhanças dos termos telemedicine, telehealth e telecare (do inglês, telemedicina, telessaúde e teleatendimento). Apesar dessas discussões, todos esses termos envolvem a transferência de informações sobre questões relacionadas à saúde entre um ou mais locais, para que a saúde dos indivíduos e de suas comunidades possa ser promovida (CRAIG; PATTERSON, 2005).

Frequentemente os termos telemedicine, telehealth e telecare são utilizados de forma intercambiável, embora possam existir diferenças de significados entre elas. $\mathrm{O}$ termo telemedicine designa o uso de TIC para apoiar a prestação de todos os tipos de serviços médicos, diagnósticos e relacionados ao tratamento, geralmente por médicos. O telehealth se assemelha a telemedicine, porém, de forma mais ampla, pode incluir uma variedade de serviços remotos de saúde, além da relação médico-paciente, envolvendo, inclusive, outros serviços prestados por enfermeiros, farmacêuticos e assistentes sociais, por exemplo. Já o termo telecare se refere à tecnologias que permitem que consumidores fiquem seguros e independentes em suas próprias casas, incluindo aplicativos de saúde e condicionamento físico, sensores e outras ferramentas que conectam os consumidores a membros da família ou outros cuidadores, ferramentas de rastreamento, sistemas de lembrete ou tecnologias de alerta e detecção precoce (FCC, 2014). 
A telemedicina é uma modalidade comprovada para fornecer cuidados paliativos às pessoas mais vulneráveis. O cuidado de pessoas com doenças graves e suas famílias agora requer distanciamento social impecável para sua proteção e para todos os profissionais de saúde tão necessários em resposta à pandemia de Covid-19 (CALTON; ABEDINI; FRATKIN, 2020). A telemedicina tem sido a primeira linha de defesa dos médicos para retardar a disseminação do coronavírus, dando suporte aos sistemas de saúde, especialmente o da rede pública, prevenção e práticas clínicas, contribuindo com o distanciamento social, fornecendo serviços por telefone ou videoconferência para cuidados pessoais leves para que os suprimentos limitados estejam focados nos casos mais urgentes (VIDAL-ALABALL et al., 2020).

Na visão de Rockwell e Gilroy (2020), os sistemas de telemedicinas podem ser ideais para amenizar superlotações em hospitais e clínicas, isso por meio da triagem de pacientes de baixa acuidade, além de evitar exposições humanas desnecessárias e promover a prestação de cuidados de alta qualidade. Nos últimos anos, leis e regulamentações de nível estadual, federal e internacional se expandiram para acomodar uma maior adoção desses sistemas de telemedicina. Para Ohannessian (2015), o uso da telemedicina em situações epidêmicas tem alto potencial para melhorar as investigações epidemiológicas, o controle de doenças e o gerenciamento de casos clínicos, ao mesmo tempo em que permite que a comunidade global de saúde apoie os cuidadores locais. Mais pesquisas seriam necessárias para obter uma melhor compreensão de como a telemedicina poderia ser aplicada ainda mais em situações de epidemia.

Entendendo a importância dessa temática, este estudo tem o objetivo de realizar uma prospecção de tecnologias de medicina remota, interpretando os dados obtidos na base de patentes como fonte de informação tecnológica. A expectativa é promover uma apresentação do panorama das tecnologias relacionadas à telemedicina, analisando a evolução temporal da tecnologia, aplicada nos principais países e empresas detentores dessas tecnologias, as áreas tecnológicas por Classificação Internacional de Patentes (CIP) e a situação do Brasil quanto a patentes de telemedicina.

\section{Metodologia}

Este trabalho realizou uma prospecção tecnológica utilizando base de patentes como fonte de informação acerca da produção tecnológica da telemedicina no mundo. O sistema de inteligência Questel Orbit foi escolhido como a base de patentes desde estudo. O Questel Orbit é uma plataforma que reúne publicações de patentes dos principais bancos de patentes do mundo, incluindo documentos de patentes de 87 escritórios nacionais e seis escritórios regionais (EPO, WIPO, OAPI, ARIPO, EAPO e CGC), além de possuir ferramentas robustas que possibilitam diversas análises de informações contidas nos documentos de patente.

Foram definidas as palavras-chave telemedicine, telehealth e telecare, que, apesar das diferenças que existem entre elas, em geral, essas palavras remetem o objeto de estudo a ser investigado. Para compor a estratégia de busca, esses termos foram combinados utilizando o operador booleano "OR". A string foi aplicada considerando os campos título, resumo e reivindicações dos documentos de patentes no sistema Orbit. As buscas foram realizadas entre os meses de setembro a novembro de 2020. A estratégia de busca é apresentada no Quadro 1. 
Quadro 1 - Estratégia de busca de patentes

\begin{tabular}{|c|c|}
\hline String & CAMPOS APLICAdos no ORBIT \\
\hline "telemedicine" OR "telehealth" OR “telecare” & Título, Resumo e Reivindicações \\
\hline
\end{tabular}

Fonte: Dados da pesquisa

Com o resultado apurado, análises foram realizadas utilizando as próprias ferramentas de inteligência do Orbit, como a evolução temporal dos pedidos de patentes, o status legal, a abrangência geográfica, os principais requerentes, as classificações das patentes por código CIP e a relação de patentes com prioridade brasileira.

\section{Resultados e Discussão}

Aplicando a metodologia descrita anteriormente, as palavras-chave utilizadas neste estudo foram testadas isoladamente para se compreender a magnitude de cada uma delas. Conforme é apresentado na Figura 1, a palavra telemedicine retornou um total de 1.456 famílias de patentes, a palavra telehealth retornou 109 familias de patentes e a palavra telecare resultou 54 famílias de patentes. Interseccionando esse resultado, por meio da string "telemedicine OR telecare OR telehealth", foram apuradas 1.523 famílias de patentes, que foram utilizadas para as análises deste estudo.

Figura 1 - Resultado das strings aplicadas na base de patentes Orbit

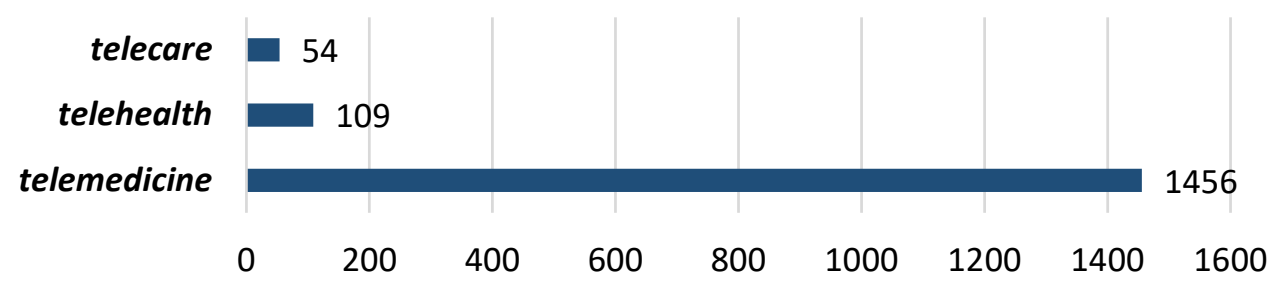

Fonte: Dados da pesquisa

Conhecer o status legal de patentes pode ser importante para compreender o atual panorama de determinados domínios tecnológicos quanto à sua situação jurídica. A Figura 2 apresenta o atual status legal das patentes de telemedicina. Desse resultado, 29,7\% das patentes já foram concedidas, $29,7 \%$ estão pendentes, $10,6 \%$ estão revogadas, $28,4 \%$ estão caducadas e $1,6 \%$ já expiraram. 
Figura 2 - Status legal das patentes levantadas

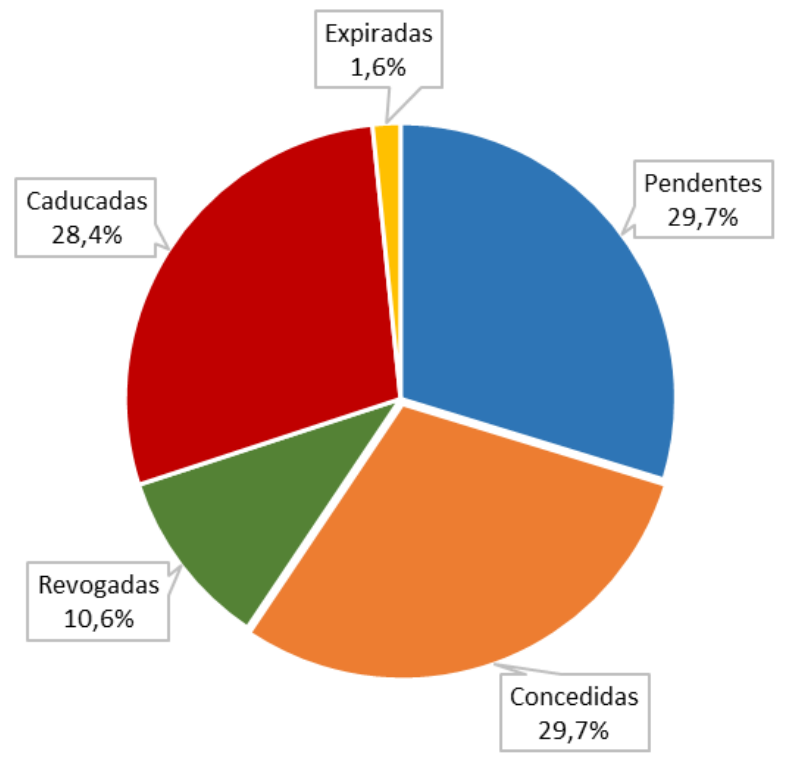

Fonte: Dados da pesquisa

Percebe-se certa proporcionalidade nessa distribuição de patentes quanto ao seu status legal, em especial a patentes concedidas, pendentes e caducadas. Nesse caso, mais da metade das patentes (pendentes e concedidas) podem estar disponíveis para exploração de mercado, levando em consideração que muitas patentes podem começar a ser exploradas após o seu depósito. Já as patentes caducadas, que representam cerca de um terço do apurado neste estudo, representam tecnologias que perderam o título pelo não uso da patente ou por possíveis abusos na sua exploração.

A Figura 3 apresenta a evolução temporal das patentes de telemedicina, considerando o ano de primeiro depósito $e$ o ano de publicação. A primeira patente que se tem registro foi depositado em 1992, intitulada "Dispositivo de atendimento de chamada de imagem para arquivos centrais sem papel”, de titularidade da então empresa francesa de telecomunicações Alcatel Sel.

Figura 3 - Evolução temporal dos pedidos de patentes por ano de primeiro depósito e ano de publicação

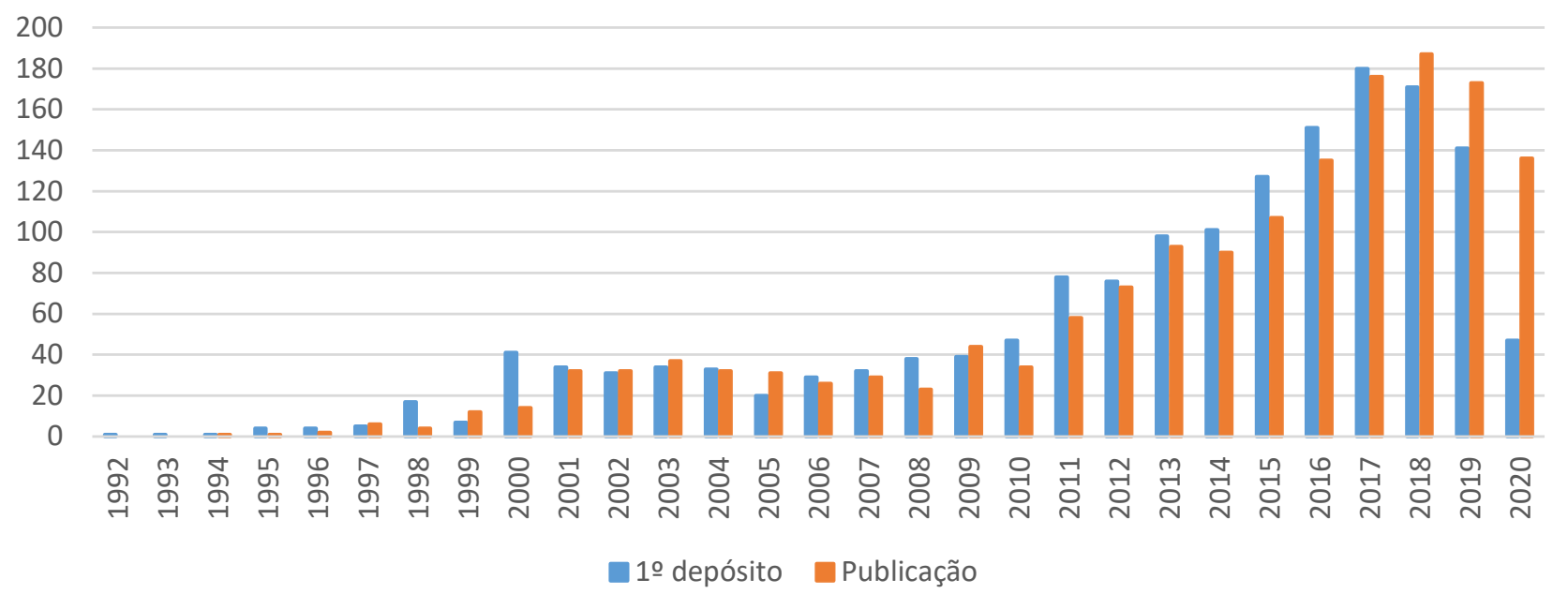

Fonte: Dados da pesquisa 
Apenas no ano 2000 que essas tecnologias começaram a ter um investimento maior em termos de depósitos de patentes, porém é possível notar que houve certa estabilidade nessa década. Já, a partir do ano de 2010, é possível notar que o crescimento desses depósitos e publicações tiveram um crescimento considerável, sendo 2017 o ano com o maior número de famílias de patentes depositadas e 2018 o ano com maior número de famílias de patentes publicadas. Apesar do decrescimento no número de depósitos nos anos de 2019 e 2020, é provável que os dados estivessem incompletos, tendo em vista os prazos protocolares nos diversos escritórios de patentes, que, em geral, demandam tempo para publicitar as informações devido, principalmente, ao período de sigilo que é imposto à patente após o seu depósito. $\mathrm{O}$ avanço tecnológico das atuais TICs, que incluem principalmente as tecnologias móveis, pode ter sido o grande motor para o forte investimento patentário que se deu nessa última década nas tecnologias de telemedicina.

De acordo com a OMS (2016), considerando o ano de 1990 como ponto de partida, houve uma evolução considerável no número de países que começaram a adotar políticas de Cobertura de Saúde Universal (UHC), E-health (saúde eletrônica), Sistema de Informação em Saúde (Health Information System - HIS) e de Telessaúde. Para Bohlin et al. (2017), nesta última década, previsões já apontavam para um crescimento anual nos investimentos em tecnologias de digitalização para o setor da saúde, entre os anos de 2013 a 2020. Essas tecnologias abrangem desde os registros médicos eletrônicos e gerenciamento de dados de pacientes até sistemas mais complexos que abrangem biossensores, dispositivos vestíveis (wereables), dispositivos móveis, que integram sistemas de telemedicina.

Com isso, o rápido crescimento da medicina a distância, apoiada pelos avanços tecnológicos, tornou esse serviço um valor agregado, segundo especialistas na indústria de telemedicina $e$ nos mercados globais de saúde, tornando-se parte integrante do apoio aos pacientes e consumidores de saúde. Além disso, há muitos estudos apontando uma alta satisfação de pacientes com a experiência da telemedicina (WALLER; STOLER, 2018), o que de fato pode demandar e estimular o desenvolvimento de inovações para esse setor.

Em 2016, o mercado global de tecnologias de telemedicina foi avaliado em 26,7 bilhões de dólares com previsão de alcançar 77,2 bilhões em 2022, crescendo a um CAGR de 19,4\%, durante esse período (2016-2022) (BUSINESS RESEARCH COMPANY, 2018). Com tudo, diante da atual crise causada pela pandemia da Covid-19, que impactou consideravelmente o mercado da telemedicina, muitas previsões foram revisadas. Em 2019, o tamanho desse mercado já era de 41,63 bilhões de dólares, novos estudos já apontam previsões de esse mercado alcançar 396,76 bilhões em 2027, a um CAGR de 25,8\% no período de 2020-2027. A adoção de novas políticas e diretrizes de telemedicina por meio de órgãos governamentais tem sido a grande chave para estreitar as barreiras para a adoção de tecnologias de telemedicina, o que poderá abrir mais oportunidade de crescimento para o mercado (FORTUNE BUSINESS INSIGHT, 2020).

O gráfico da Figura 4 apresenta o número de famílias de patentes por país de prioridade, considerando os 20 principais. É possível notar 17 nações, além dos escritórios da World Intellectual Property Organization (WIPO) e do European Patent Office (EPO) que também estão incluídos nessa análise. 
Figura 4 - Quantitativo de depósitos de patentes por país de prioridade

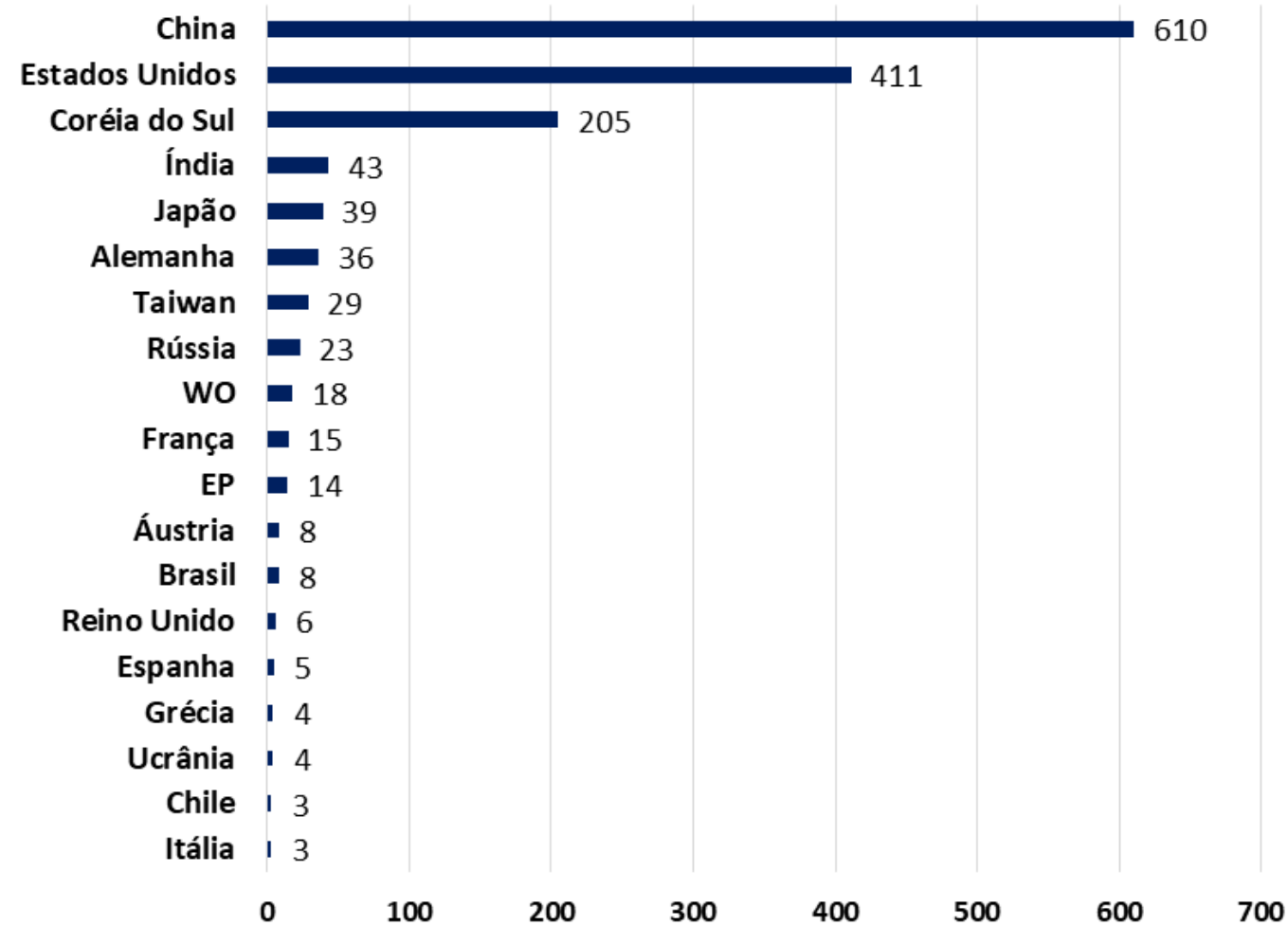

Fonte: Dados da pesquisa

A China aparece na primera posição com 610 famílias de patentes, seguida pelos Estados Unidos com 411 depósitos e a Coreia do Sul apresenta 205 famílias de patentes depositadas. Os demais países aparecem com menos de 50 famílias de patentes cada, entre eles a Índia com 43 depósitos, o Japão com 39 patentes, a Alemanha com 36 patentes e o Brasil com com oito famílias de patentes. A produção patentária da China, dos Estados Unidos e da Coreia do Sul representa cerca de $80 \%$ de todo levantamento patentário apurado neste estudo.Um dos pilares centrais da nova reforma da saúde na China é a chamada Intelligent Healthcare (saúde inteligente), que tem recebido grandes quantias de investimentos de recursos para o seu desenvolvimento, a expectativa do país é solucionar um dos seus principais conflitos, recursos limitados de assistência médica e grande população de pacientes (ZHENG; RODRIGUEZ-MONROY, 2015). O mercado da saúde na China cresceu a uma taxa consistentemente rápida nos últimos cinco anos e, em 2019 , atingiu \$1,1 trilhão de dólares, um aumento de 10\% em comparação ao ano anterior. A China que foi o primeiro epicentro da Covid-19 presenciou uma rápida aceleração da adoção generalizada da tecnologia digital, tanto na prestação de serviços de saúde (monitoramento remoto de pacientes, reserva on-line e telemedicina) quanto na entrega assistida digitalmente em serviços de saúde (inteligência artificial, aprendizado de máquina para diagnóstico e tratamento, realidade aumentada ou treinamento de cirurgiões de RA, robótica e assistência de IA). Além disso, a adoção do 5G e da Internet das Coisas (IoT) no suporte à assistência médica digitalizada e na telemedicina continuará colocando essas áreas no foco de planejadores governamentais nos próximos anos (CHINA BRIEFING, 2020). 
Apesar de o resultado apresentar os Estados Unidos em segundo lugar no ranking de patentes de telemedicina, o país é considerado líder de mercado em serviços de telessaúde. A Europa caminha para se tornar o segundo maior mercado, enquanto a região da Ásia-Pacífico é a região que tem apresentado o crescimento mais rápido no mercado global, devido principalmente ao aumento da população geriátrica, visto que pequenas empresas estão procurando maneiras de reduzir os custos de saúde e, ao mesmo tempo, atender melhor a população idosa e aqueles que sofrem de doenças crônicas (INTOUCH HEALTH, 2021). Já a Coreia do Sul, onde a telemedicina era permitida apenas para fins experimentais, presenciou suas legislações proibitivas de telemedicina serem flexibilizadas diante da crise global da Covid-19 (KOREA HERALD, 2020). Desde 2017, o governo tem identificado a saúde digital como uma área fundamental para o crescimento da Coreia do Sul. Além disso, a tecnologia $5 \mathrm{G}$ já está bastante disseminada no país, tornando a Coreia um mercado forte em telecomunicações na área da saúde digital (STL PARTNERS, 2017).

A Tabela 1 apresenta o ranking com as dez principais empresas e instituições requerentes de patentes de telemedicina, sendo um excelente indicador de inventividade dos principais competidores ativos no mercado. Apesar do expressivo número de patentes chinesas apontado anteriormente, é apresentada nesse ranking uma considerável diversidade de países em que as referentes empresas estão localizadas.

Tabela 1 - Ranking de empresas requerentes de patentes e sua localização geográfica

\begin{tabular}{ccc} 
EMPRESA/INSTITUIÇÃo & Famílias de PateNtes & País \\
PHILLIPS & 13 & Holanda \\
ROBERT BOSCH & 11 & Alemanha \\
SAMSUNG ELETRONICS & 10 & Coreia do Sul \\
SIEMENS & 9 & Alemanha \\
ANYCHECK INFORMATION TECHNOLOGY & 6 & China \\
BOE TECHNOLOGY & 6 & China \\
INTOUCH TECHNOLOGIES & 6 & Estados Unidos \\
NANJING UNIVERSITY OF INFORMATION & 6 & China \\
SCIENCE \& TECHNOLOGY & & \\
\hline
\end{tabular}

Fone: Dados da pesquisa

É possível notar nesse ranking empresas e instituições de ao menos seis países, sendo a China o país com o maior número de empresas. Porém, a primeira posição é ocupada pela holandesa Phillips com 13 famílias de patentes de telemedicina. A multinacional alemã de engenharia eletrônica Robert Bosch aparece na segunda posição com 11 famílias de patentes. A compania sul-coreana Sansung Eletronics ocupa a terceira posição nesse ranking com 10 famílias de patentes de telemedicina. O conglomerado industrial alemão Siemens aparece na quarta posição com nove famílias de patentes. Há mais de três décadas, a Philips é líder em telessaúde e em configurações de cuidados agudos e pós-agudos no hospital e em casa, ajudando sistemas de saúde a implantarem e a dimensionarem soluções de telessaúde para ajudar a melhorar os resultados do paciente, aprimorar a experiência do paciente e da equipe, além de 
diminuir os custos no atendimento. Recentemente, a Philips recebeu autorização da Food and Drug Administration para seu biossensor portátil sem fio (Philips Biossensor BX100) para ajudar a gerenciar pacientes com Covid-19 confirmados e suspeitos no hospital. O biossensor vestível aprimora a vigilância clínica como parte da solução de detecção de deterioração do paciente para ajudar os médicos a detectarem o risco, para que possam intervir mais cedo e ajudar a melhorar o atendimento aos pacientes em áreas de baixa acuidade (GLOBENEWSWIRE, 2020).

A Robert Bosch também se destacou na última década como líder de mercado em soluções de telessaúde. A empresa conhecida pela sua ferocidade na defesa de sua propriedade intelectual desde 2015 resolveu mudar sua estratégia no mercado de telessaúde, centrando-se especificamente em tecnologias de sensores. Em um mercado com fortes concorrentes, desde 2015, a Bosch tem realinhado seu negócio no setor médico e de saúde, no intuito de examinar suas opções no mercado (MOBIHEALTH NEWS, 2015).

A gigante sul-coreana Samsung, atuando em mais de 100 países, também tem travado batalhas judiciais dentro do mercado da saúde digital (TECHRADAR, 2020). Nessa corrida pela vantagem competitiva, a empresa tem formado importantes parcerias com empresas locais em diversos países (AMWELL, 2017). Recentemente, a empresa lançou o seu aplicativo de monitoramento de pressão arterial sem presilha e sem braçadeira Samsung Health Monitor App. O aplicativo permite que os usuários verifiquem a pressão arterial usando um smartwatch e tem o potencial de atrapalhar o mercado de monitores de pressão arterial (BPM) do país. Isso também ajudará a Samsung a competir com arquirrivais como a Apple (BECKER'S HOSPITAL REVIEW, 2018).

A Siemens Healthineers está continuamente desenvolvendo seu portfólio de produtos e serviços, com aplicativos suportados por inteligência artificial e ofertas digitais que desempenham um papel cada vez mais importante na próxima geração de tecnologia médica. Com seu software Teamplay my Care Companion, a empresa está trazendo ao mercado uma nova e flexível solução de telemedicina que possibilita o atendimento remoto a pacientes com doenças crônicas e Covid-19 (DIGITAL HEALTH NEWS, 2020).

A diversidade de empresas e de países com patentes de telemedicina apontada neste estudo mostra que o mercado tecnológico da telemedicina tem se mostrado muito ativo nos últimos anos, com fortes competidores multinacionais, os quais têm se mostrado enérgicos na proteção patentária de suas tecnologias, indicando a alta concorrrência existente nesse setor.

A Classificação Internacional de Patentes (CIP) é uma excelente ferramenta para identificar o conteúdo técnico dos documentos de patentes. A Figura 5 apresenta os dez principais códigos CPI das famílias de patentes analisadas neste estudo, sendo uma boa maneira de destacar as principais áreas técnicas desse conjunto de documentos de patentes. No Quadro 2 é apresentada a descrição dos principais códigos destacados.

O código A61B-005, referente à detecção, medição e registro para fins de diagnóstico, identificação de pessoas, apresentou o maior número de famílias de patentes, com 534 famílias de patentes, seguido do grupo G06F-019, relacionado a tecnologias de equipamentos e métodos de computação digital ou processamento de dados, especialmente adaptados para aplicações específicas, com 375 famílias de patentes. O grupo G06Q-050 também se destacou nesse levantamento, relativo à sistema ou a métodos especialmente adaptados para setores de negócios específicos, este grupo apresentou um total de 282 famílias de patentes. 
Figura 5 - Quantitativo de patentes por principais códigos da CIP

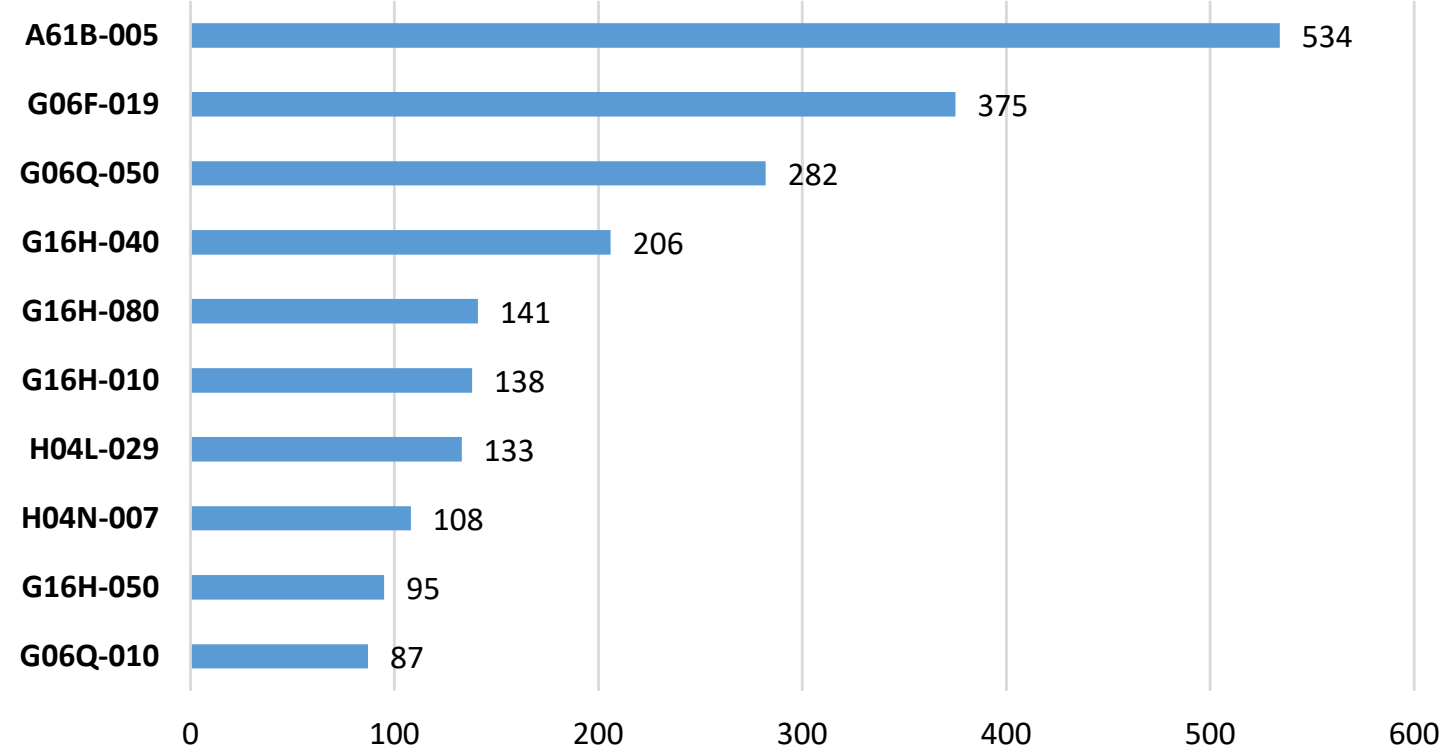

Fonte: Dados da pesquisa

Também se destacaram nesse levantamento quatro subgrupos da família de código G16H, que se referem às TICs especialmente adptadas para fins específicos, sendo elas: para a gestão ou administração de recursos ou instalações de saúde (G16H-040) com 206 famílias de patentes; para facilitar a comunicação entre médicos ou pacientes (G16H-080) com 141 famílias de patentes; para o manuseio ou processamento de dados médicos ou de saúde relacionados ao paciente (G16H-010) com 138 famílias de patentes; e para diagnóstico médico, simulação médica ou mineração de dados médicos (G16H-050) com 95 famílias de patentes.

Quadro 2 - Descrição dos principais códigos da CIPs

\begin{tabular}{|c|c|}
\hline CÓDIGO CIP & DESCRIÇÃO \\
\hline A61B-005 & Deteç̧ão, medição e registro para fins de diagnóstico, identificação de pessoas. \\
\hline G06F-019 & $\begin{array}{l}\text { Equipamentos e métodos de computação digital ou processamento de } \\
\text { dados, especialmente adaptados para aplicações específicas. }\end{array}$ \\
\hline G06Q-050 & Sistema ou métodos especialmente adaptados para setores de negócios específicos. \\
\hline G16H-040 & $\begin{array}{l}\text { TIC especialmente adaptada para a gestão ou administração de } \\
\text { recursos ou instalações de saúde, TIC especialmente adaptada para a } \\
\text { gestão ou operação de equipamentos ou dispositivos médicos. }\end{array}$ \\
\hline G16H-080 & TIC especialmente adaptada para facilitar a comunicação entre médicos ou pacientes. \\
\hline G16H-010 & $\begin{array}{l}\text { TIC especialmente adaptada para o manuseio ou processamento } \\
\text { de dados médicos ou de saúde relacionados ao paciente. }\end{array}$ \\
\hline H04L-029 & Arranjos, aparelhos, circuitos ou sistemas. \\
\hline H04N-007 & Sistemas de televisão. \\
\hline G16H-050 & $\begin{array}{l}\text { TIC especialmente adaptada para diagnóstico médico, simulação médica } \\
\text { ou mineração de dados médicos, TIC especialmente adaptada para } \\
\text { detectar, monitorar ou modelar epidemias ou pandemias. }\end{array}$ \\
\hline G06Q-010 & Gestão, administração. \\
\hline
\end{tabular}

Fonte: Dados da pesquisa 
A Figura 6 apresenta a evolução do número de depósitos de patentes dos principais códigos CIPs. Apesar de os códigos A61B-005 e G06F-019 concentrarem o maior número de patentes desse ranking, é possível notar que os códigos do grupo $\mathrm{G} 16 \mathrm{H}$, em especial o $\mathrm{G} 16 \mathrm{H}-040$ e G16H-080, que se referem a TICs adaptadas, foram os códigos de patentes em que os pedidos de depósito mais cresceram nos últimos cinco anos. De fato, o forte avanço das TICs nos últimos anos pode ter impulsionado ainda mais o desenvolvimento de tecnologias de telemedicina baseadas em TICs.

Figura 6 - Evolução temporal dos pedidos de depósito de patentes por principais códigos da CIP

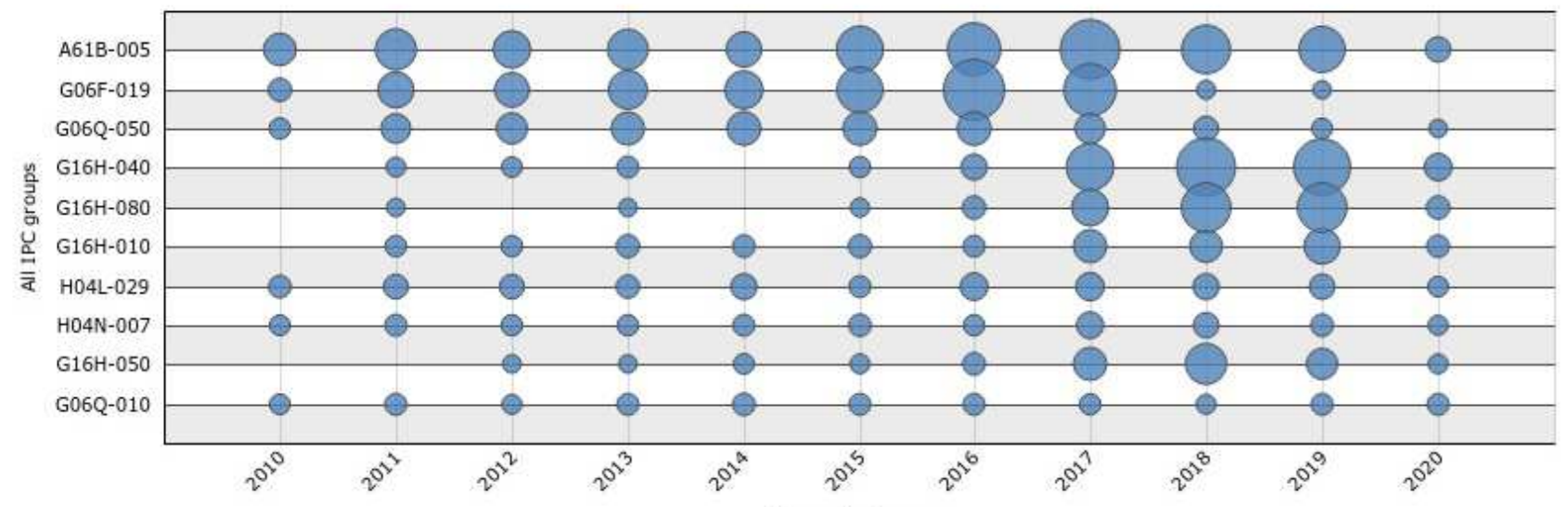

1st application year

Fonte: Dados da pesquisa

De acordo com a Anixter (2018), esses avanços têm acontecido não só pelas inovações na medicina, mas também têm ocorrido com novos equipamentos médicos e métodos de atendimento. Esses equipamentos têm utilizado cada vez mais tecnologias e dados conectados, a fim de melhor analisar e prestar assistência. Como exemplo, essas inovações têm acontecido por meio dos tradicionais prontuários eletrônicos, possibilitando que prestadores de cuidados médicos possam compartilhar um maior volume de dados médicos com mais partes interessadas para mais propósitos, que incluem diagnóstico entre pacientes e populações; vestíveis e inovações em biotelemetria, monitorar constantemente os pacientes, estejam eles ativos ou não em uma instalação, produtos de consumo como Apple Watch e o Fit Bit tornaram-se quase comuns; atendimento virtual, o atendimento virtual pode facilitar a eficiência e a qualidade do atendimento, permitindo que indivíduos que estão dentro de uma organização possam buscar a opinião de um especialista em uma instalação diferente, ou os pacientes, em casa, realizem um check-up ou diagnóstico rápido.

Das patentes apuradas neste estudo, apenas oito delas possuem prioridade brasileira, conforme é apresentado no Quadro 3. Desse resultado, três foram requeridas por universidades (Universidade Federal do Paraná, Universidade Federal de Goiás e Universidade Estadual de Campinas/Universidade Estadual do Oeste do Paraná); quatro foram requeridas por inventores independentes, sendo três deles pelos escritórios internacionais, e apenas uma foi requerida por uma empresa (Telcor Telemedicina). O depósito mais recente foi requerido pela Universidade Federal do Paraná em maio de 2018, com título "Sistema de telemedicina por cabine para detecção e descarga de distúrbios de doenças". 
Quadro 3 - Lista de patentes de telemedicina com prioridade brasileira

\begin{tabular}{|c|c|c|c|c|}
\hline \# & Código & Título & REQUERENTE & $\begin{array}{l}\text { DATA DE } \\
\text { PRIORIDADE }\end{array}$ \\
\hline $\mathbf{1}$ & BR102018009083 & $\begin{array}{l}\text { Sistema de telemedicina por cabine para } \\
\text { detecção e descarga de distúrbios de doenças. }\end{array}$ & $\begin{array}{l}\text { Universidade } \\
\text { Federal do } \\
\text { Paraná }\end{array}$ & 04/05/2018 \\
\hline 2 & BR102018002540 & $\begin{array}{l}\text { Telemedicina para compartilhamento } \\
\text { de informações e monitoramento } \\
\text { de pacientes a distância. }\end{array}$ & $\begin{array}{l}\text { Inventores } \\
\text { independentes }\end{array}$ & 06/02/2018 \\
\hline 3 & BR102015030674 & $\begin{array}{l}\text { Biossensor calorímetro integrado na } \\
\text { plataforma de papel de monitoramento } \\
\text { de glicose em amostras de lágrima. }\end{array}$ & $\begin{array}{l}\text { Universidade } \\
\text { Federal de Goiás }\end{array}$ & 08/12/2015 \\
\hline 4 & EP3131030 & $\begin{array}{l}\text { Centro de controle de inteligência } \\
\text { artificial médica com sistema remoto para } \\
\text { elaboração de diagnósticos, prescrição } \\
\text { de medicamentos e envio de tratamento } \\
\text { médico on-line via telemedicina. }\end{array}$ & $\begin{array}{l}\text { Inventores } \\
\text { independentes }\end{array}$ & $10 / 08 / 2015$ \\
\hline 5 & EP3253022 & $\begin{array}{l}\text { Sistema, equipamento e método } \\
\text { para realizar e documentar em tempo } \\
\text { real um procedimento profissional } \\
\text { assistido remotamente. }\end{array}$ & $\begin{array}{l}\text { Inventores } \\
\text { independentes }\end{array}$ & $28 / 01 / 2015$ \\
\hline 6 & WO2014/094095 & $\begin{array}{l}\text { Método de telemedicina para } \\
\text { monitoramento remoto em tempo } \\
\text { real de procedimentos médicos. }\end{array}$ & $\begin{array}{l}\text { Universidade } \\
\text { Estadual de } \\
\text { Campinas/ } \\
\text { Universidade } \\
\text { Estadual do } \\
\text { Oeste do Paraná }\end{array}$ & $21 / 12 / 2012$ \\
\hline 7 & BRPI1005462 & Sistema de Telemedicina. & $\begin{array}{c}\text { Telcor } \\
\text { Telemedicina }\end{array}$ & $21 / 12 / 2010$ \\
\hline 8 & WO2008/022423 & $\begin{array}{l}\text { Sistema de telemedicina para } \\
\text { monitoramento remoto de pacientes. }\end{array}$ & $\begin{array}{l}\text { Inventores } \\
\text { independentes }\end{array}$ & $25 / 08 / 2006$ \\
\hline
\end{tabular}

Fonte: Dados da pesquisa

Apesar da produção ínfima do Brasil de tecnologias de telemedicina, considerando seu número de patentes levantadas neste estudo, desde 2006, algumas importantes iniciativas vêm ocorrendo no processo de desenvolvimento da telemedicina no país, como a Rede Universitária de Telemedicina (RUTE), o Programa Nacional de Telessaúde na Atenção Primária e o Acordo Internacional Internet2 e RNP, no desenvolvimento da próxima geração de tecnologias e aplicações Internet, Telemedicina e Telessaúde, RUTE e US Health Sciences (MESSINA et al., 2012). Entretanto, para que o país atinja seu pleno desenvolvimento, os desafios ainda são pertinentes, levando em consideração que a telemedicina possui uma natureza interdisciplinar, o que significa que seu desenvolvimento requer a convergência de diversas áreas do conhecimento - médica, TIC, microeletrônica, informática, telecomunicações, equipamentos, entre outras -, o que corrobora com a necessidade de uma visão sistêmica e de ações conjuntas e coordenadas entre diferentes instâncias decisórias, com a participação da indústria, da academia, de instituições científicas e tecnológicas, de associações de classe, entre outros agentes relevantes no processo de inovação (MALDONADO; MARQUES; CRUZ, 2016). 
Diante da crise da Covid-19, muitas iniciativas também têm se proliferado pelo país. Em abril de 2020, foi publicada a Lei n. 13.989, que dispõe sobre o uso da telemedicina durante a crise causada pelo coronavírus (BRASIL, 2020). Para Silva et al. (2020), a atual política de telemedicina brasileira está em processo de aprimoramento, uma vez que o conjunto de prioridades e consensos sobre como realizar as atividades relacionadas ainda é objeto de discussão e disputa por diversos grupos de interesse, internos e externos ao Ministério da Saúde. Entre essas iniciativas, destaca-se a implementação da rede TeleICU Respiratória, que tem sido uma abordagem de alto nível para gerenciar a pandemia causada pelo SARS-CoV -2. Essa rede permitiu o estabelecimento de um protocolo de gestão e facilitou a formação e a supervisão de mais de 19 equipes de referência nesse serviço(CARVALHO et al., 2020).

\section{Considerações Finais}

Este artigo teve o objetivo de apresentar um estudo de prospecção de tecnologias de telemedicina utilizando informações de patentes. Foi observado que houve considerável crescimento no número de depósitos de patentes de telemedicina, em especial a partir do ano 2010. Quase $60 \%$ dos depósitos apurados se tratam de patentes concedidas ou patentes em fase de concessão.

Verificou-se que China, Estados Unidos e Coreia do Sul são as nações mais atuantes na atividade patentária envolvendo tecnologias de telemedicina. Já o ranking de requerentes de patentes de telemedicina destacou importantes multinacionais, como Philips, Bosch e Samsung, empresas em competição ativa no mercado de tecnologias de medicina remota.

Apesar de surgirem importantes iniciativas em direção ao desenvolvimento da telemecina no Brasil, o país ainda possui uma produção ínfima de tecnologias de telemedicina frente a outras nações, $e$, considerando seu número de patentes, que em sua maioria foi protegida por universidades públicas.

Notou-se um relevante número de tecnologias classificadas na CIP A61B-005, que se referem a tecnologias de detecção, medição e registro para fins de diagnóstico. Também foi observado que nos últimos cinco anos houve forte tendência de tecnologias do grupo G16H, que se referem a TICs especialmente adptadas para fins específicos.

Para mais, este estudo mostrou que a área da telemedicina cresceu consideravelmente nos últimos anos, com fortes competidores no mercado e com o investimento de grandes potencias mundiais. O forte avanço das TICs, em especial as tecnologias móveis, é um dos principais propulssores do desenvolvimento dessas tecnologias, principalmente nos últimos anos.

\section{Perspectivas Futuras}

Com a crise sanitária causada pela pandemia da Covid-19, a área da telemedicina ganhou mais evidência por parte de empresas que desenvolvem tecnologias e de prestadores de serviços de saúde que se utilizam dessas tecnologias. Isso já tem impulsionado esse mercado, e as estimativas são de forte crescimento nos próximos anos. Espera-se que este estudo ajude a nortear tomadores de decisões ligados à área da saúde, equipes de assistência médica e pesquisadores e, também, ajude no desenvolvimento de pesquisas mais específicas relacionadas a tecnologias de medicina remota. 


\section{Referências}

AMWELL - AMERICAN WELL. American Well Telehealth Now Live on New Samsung Health. 2017. Disponível em: https://business.amwell.com/press-release/american-well-telehealth-now-liveon-new-samsung-health/. Acesso em: 15 jan. 2021.

ANIXTER. Global Technology Briefing Smart Hospital Infrastructure Best Practices. [S.l.]: Anixter Inc. 2018.

BECKER'S HOSPITL REVIEW. Samsung enters UK telehealth market through Babylon partnership. 2018. Disponível em: https://www.beckershospitalreview.com/telehealth/samsungenters-uk-telehealth-market-through-babylon-partnership.html $+\& \mathrm{~cd}=1 \& \mathrm{hl}=\mathrm{pt}-\mathrm{BR} \& \mathrm{ct}=\mathrm{clnk} \& \mathrm{gl}=\mathrm{br}$. Acesso em: 15 jan. 2021.

BOHLIN, N. et al. Building the Smart Hospital Agenda - A comprehensive approach for Hospitals executives to develop their Smart Hospital Strategy and Implementation program. [S.I.]: Arthur D. Little, 2017.

BRASIL. Lei n. 13.989, de 15 de abril de 2020. Dispõe sobre o uso da telemedicina durante a crise causada pelo coronavírus (SARS-CoV-2). Diário Oficial da União, Brasília, DF, 16 de abril de 2020. Disponível em: https://www.in.gov.br/en/web/dou/-/lei-n-13.989-de-15-de-abrilde-2020-252726328. Acesso em: 16 fev. 2021.

BUSINESS RESEARCH COMPANY. Telemedicine Technologies Market - By Type (Tele-Home \& Tele-Hospital), By Applications (Tele-Radiology, Tele-Consultation, Tele-Monitoring and TeleSurgery), and By Region, Opportunities And Strategies - Global Forecast To 2022. Relatório de Pesquisa Mercado, [s.l.], 2018. Disponível em: https:/www.thebusinessresearchcompany.com/ report/telemedicine-technologies-market. Acesso em: 15 jan. 2021.

KOREA HERALD, S. Korea adopts telemedicine to battle coronavirus outbreak. 2020. Disponível em: http://www.koreaherald.com/view.php?ud=20200313000725. Acesso em: 15 jan. 2021.

CALTON, B.; ABEDINI, N.; FRATKIN, M. Telemedicine in the Time of Coronavirus. J Pain Symptom Manage, [s.l.], v. 60, n. 1, p.12-14, 2020.

CARVALHO, C. R. R. et al. Use of telemedicine to combat the COVID-19 pandemic in Brazil. Clinics, [s.l.], v. 75, 2020.

CHINA BRIEFING. China's Healthcare Industry - Opportunities in Telemedicine and Digital Healthcare. 2020. Disponível em: https://www.china-briefing.com/news/china-investment-outlooktelemedicine-digital-healthcare-industry/. Acesso em: 15 jan. 2020.

CRAIG, J.; PATTERSON, V. Introduction to the practice of telemedicine. J Telemed Telecare, [s.l.], v. 11, n. 1, p. 3-9, 2005.

DIGITAL HEALTH NEWS. Siemens Healthineers Offers Flexible Telemedicine Solution for Healthcare Providers. 2020. Disponível em: https://www.digitalhealthnews.eu/siemens/6168siemens-healthineers-offers-flexible-telemedicine-solution-for-healthcare-providers. Acesso em: 15 jan. 2021.

FCC - FEDERAL COMMUNICATIONS COMMISSION. Telehealth, Telemedicine, and Telecare: What's What? 2014. Disponível em: https://www.fcc.gov/general/telehealth-telemedicine-andtelecare-whats-what. Acesso em: 15 jan. 2021. 
FORTUNE BUSINESS INSIGHT. Telemedicine Market Size, Share \& COVID-19 Impact Analysis. Relatório de pesquisa de Mercado, [s.l.], 2021.

GLOBEWSWIRE. Philips announces collaboration with American Telemedicine Association to further commitment to telehealth. 2020. Disponível em: https://www.globenewswire. com/news-release/2020/06/18/2050157/0/en/Philips-announces-collaboration-with-AmericanTelemedicine-Association-to-further-commitment-to-telehealth.html. Acesso em: 15 jan. 2021.

KVEDAR, J.; COYE, M. J.; EVERETT, W. Connected health: a review of technologies and strategies to improve patient care with telemedicine and telehealth. Health Aff (Millwood), [s.l.], v. 33, n. 2, p. 194-199, 2014.

INTOUCH HEALTH. The Future of Telehealth in the U.S. and Across the Globe. 2021. Disponível em: https://intouchhealth.com/future-telehealth-us-across-globe/. Acesso em: 15 jan. 2021.

MALDONADO, J. M. S. V.; MARQUES, A. B.; CRUZ, A. Telemedicina: desafios à sua difusão no Brasil. Cad. Saúde Pública, Rio de Janeiro, v. 32, supl., 2016.

MESSINA, L. A. et al. A Rede Universitária de Telemedicina - RUTE. Gold Book Inovação Tecnológica em Educação e Saúde. Rio de Janeiro: EdUERJ, 2012. Disponível em: http://www. telessaude.uerj.br/goldbook/creditos. Acesso em: 16 mar. 2020.

MOBIHEALTHNEWS. Bosch shutters pioneering telehealth service Health Buddy, US-based unit. 2015. Disponível em: https://www.mobihealthnews.com/44747/bosch-shutters-pioneeringtelehealth-service-health-buddy-us-based-unit. Acesso em: 15 jan. 2021.

OHANNESSIAN, R. Telemedicine: Potential applications in epidemic situations. European Research in Telemedicine, Nota Técnica, [s.l.], v. 4, n. 3, p. 95-98, 2015.

OMS - ORGANIZAÇÃO MUNDIAL DA SAÚDE. Telemedicine - Oportunities and developments in Member States. Global Observatory for eHealth series, [s.l.], v. 2, 2010.

OMS - ORGANIZAÇÃO MUNDIAL DA SAÚDE. Global diffusion of eHealth - Making universal health coverage achievable: Report of the third global survey on eHealth. Geneva: OMS, 2016.

ROCKWELL, K. L.; GILROY, A. S. Incorporating Telemedicine as Part of COVID-19 Outbreak Response Systems. The American Journal of Managed Care, [s.l.], v. 26, n. 4, 2020.

SILVA, A. B. et al. Three decades of telemedicine in Brazil: Mapping the regulatory framework from 1990 to 2018. Plos One, [s.l.], v. 15, n. 11, 2020.

SLT PARTNERS. Digital health in South Korea: five examples of digital health beyond telemedicine. 2017. Disponível em: https://stlpartners.com/digital-health-telecoms/digital-health-insouth-korea-five-examples-of-digital-health-beyond-telemedicine/. Acesso em: 15 jan. 2021.

TECHRADAR. Samsung Health app faces a battle on patent violation. 2020. Disponível em: https://www.techradar.com/news/samsung-health-app-faces-a-battle-on-patent-violation. Acesso em: 15 jan. 2021.

THE BUSINESS RESEARCH COMPANY. Telemedicine Technologies Market - By Type (Tele-Home \& Tele-Hospital), By Applications (Tele-Radiology, Tele-Consultation, Tele-Monitoring and TeleSurgery), and By Region, Opportunities And Strategies - Global Forecast To 2022. Relatório de Pesquisa Mercado, [s.l.], 2018. Disponível em: https:/www.thebusinessresearchcompany.com/ report/telemedicine-technologies-market. Acesso em: 15 jan. 2021. 
THE KOREA HERALD. S. Korea adopts telemedicine to battle coronavirus outbreak. 2020. Disponível em: http://www.koreaherald.com/view.php?ud=20200313000725. Acesso em: 15 jan. 2021.

VIDAL-ALABALL, J. et al. Telemedicine in the face of the COVID-19 pandemic. Atención Primaria, [s.l.], v. 52, n. 6, p. 418-422, 2020. DOI: https://doi.org/10.1016/j.aprim.2020.04.003.

WALLER, M.; STOLER, C. Telemedicine: a Primer. Current Allergy and Asthma Reports, [s.l.], v. 54, n. 18, p. 1-9, 2018.

WEINSTEIN, R. S. et al. Telemedicine, telehealth, and mobile health applications that work: opportunities and barriers. Am. J. Med., [s.l.], v. 127, n. 3, p. 183-187, 2014.

DOI: 10.1016/j.amjmed.2013.09.032.

ZHENG, X.; RODRÍGUEZ-MONROY, C. The Development of Intelligent Healthcare in China.

Telemedicine and e-Health, [s.l.], v. 21, n. 5, p. 443-448, 2015.

\section{Sobre os Autores}

\section{Felipe Roberto Eloi Moura}

E-mail: felipemoura.al@gmail.com

ORCID: http://orcid.org/0000-0002-7509-1942

Mestre.

Endereço profissional: Rua Ministro Salgado Filho, n. 78, Pitanguinha, Maceió, AL. CEP: 57052-140.

\section{Kaline Silva dos Santos}

E-mail: kalinessantos@hotmail.com

ORCID: http://orcid.org/0000-0002-3599-512X

Mestre.

Endereço profissional: Av. Professor Santos Ferraz, n. 213, Poço, Residencial Maravilha, apto. 106, Bloco A, Maceió, AL. CEP: 57025040.

\section{Ailton Mota do Nascimento Galvão}

E-mail: ailton_fisioterapeuta@hotmail.com

ORCID: http://orcid.org/0000-0002-9268-6921

Mestre.

Endereço profissional: Rua Hugo Correia Paes, n. 253, Gruta de Lourdes, Maceió, AL. CEP: 57.052-827.

\section{Guilherme Benjamin Brandão Pitta}

E-mail: guilhermebbpitta@gmail.com

ORCID: http://orcid.org/0000-0003-2790-2015

Doutor.

Endereço profissional: Rua Hugo Correia Paes, n. 253, Gruta de Lourdes, Maceió, AL. CEP: 57.052-827. 